\title{
An extended digital image correlation method for mapping multiscale damage in concrete
}

\author{
$\mathrm{H}$ Mamand $^{1}$ and $\mathrm{J}$ Chen $^{2 *}$ \\ School of Civil Engineering and Surveying, Faculty of Technology \\ University of Portsmouth, Portsmouth PO1 3AH, UK
}

\begin{abstract}
This paper presents an extended digital image correlation (EDIC) method for mapping multiscale damage in concrete. The EDIC method is developed based on the distance transformation algorithm, which extends the capability of current digital image correlation (DIC) method in detecting micro damage. Applications of the EDIC in mapping multiscale damage in concrete specimens are given in this paper, which proves the capability of the EDIC in detecting multiscale damage in concrete. This novel EDIC technology can be used for further research on material damages in the society of concrete academia, and for improving safety assessment level by detecting micro damage in industrial applications.
\end{abstract}

Key words: Extended digital image correlation, Mapping damages, Distance transformation algorithm, Critical damage strain, Multiscale crack

\section{Introduction}

Damage is one of main problems in the ageing concrete, which can be seen from many concrete structures e.g. coast infrastructures. A lot of severe damage can be observed from the concrete beam surfaces. The appearance and growth of micro cracks in reinforced concrete located in coastal and offshore regions are a major concern, because these structures are frequently subjected to cyclic or tidal exposures initiating dry-wet cycles providing a constant source of salts containing chloride and sulphate ions [1,2]. These effects lessen significant serviceability, durability, and strength of reinforced concrete. It is a fact that concrete is composed of different sized aggregates, the material is heterogeneous. Therefore, the crack in concrete may be modelled at different scales. Jacobsen et al. [3] mentioned in their previous work in 2013 that the complete damages in concrete would be studied by detecting micro and macro cracks. From this point of view, the existing normal cracks are either visual macro cracks (above $1 \mathrm{~mm}$ crack width defined by this investigation), or invisible 
micro cracks (between $10 \mu \mathrm{m}$ to $1000 \mu \mathrm{m}$ crack width). Kiani and Shodja [2] suggested that micro cracks can be detected by microscopes or possible non-destructive equipment.

The previous researches [4-10] used the standard digital image correlation (DIC) method to carry out strain field measurements of a wide range of engineering materials. For instance, Dai et al. [4] used the DIC to investigate deformation behaviour in metals. The deformation behaviour of thermally stressed ceramics was investigated by Coburn and Slevin [5]. Zink et al. [6] and Lyons et al. [7] used the DIC to obtain deformation and strain on the surface of woods subjected to high temperature. Over the years (2000 to 2008) the DIC has been actively practised to a wide range of materials in the material characterization studies. The materials investigated by different researchers cited in Sutton et al. [8] included thin films, foams, mineral wool, shape memory alloys, polymers, glass, sands and many others.

Melenka and Carey [26] investigated tension behaviour in fiber reinforced cement using DIC optical measurement method. DIC Camera was used to monitor the progressive failure of the splitting tensile samples by measuring the strains at initial failure as well post failure. Srikar et al. [27] also used DIC to study the temperature effects on fiber reinforced concrete by measuring the strain field of concrete exposed to temperatures. Stress-strain curves were established from the strain measurements for the tested specimen. The stress-strain response revealed enhancement in post peak with increasing fiber dosage at all exposure temperatures. Enfedaque et al. [28] used DIC technique in the fracture test analysis of glass fibre reinforced cement (GRC). The damage pattern from the DIC output in the fracture tests of the GRC (with an addition of $25 \%$ of the thermal-treated kaolin) explained the higher fracture energy found in this admixture and is proved to be the most suitable formulation for possible future structural applications.

In relation to a damage or crack investigation, Tung and Sui [9] used the DIC for analysing damage in a cylinder pipes. The DIC was used to monitor a strain surrounding the cracks and identifying stress concentration zone in the damaged pipe. Lecompte et al. [10] used camerabased measurement for a crack prediction on concrete beam surface. The approach was based on the DIC strain-field outputs to analyse the cracks propagations based on the variations of the load-strain response between the sections of investigated beam. However, this approach cannot define the exact positions of the cracks or the crack paths. Rethore et al. [11] used the DIC to analyse situations in which discontinuities in the displacement field arise. It is based on a decomposition of the DIC displacement field output onto a regular finite element basis supported by a uniform mesh to describe accurately discontinuities region. Furthermore, Rethore et al. [12] and Chen et al. [13] used the DIC in conjunction with extended finite element method (X-FEM) to measure the full-field displacement across the crack domain. 
In further research, Rethore et al. [14] investigated the technique of 3D image correlation to allow for the measurement of 3D displacement fields in the existence of cracks. Nguyen et al. [15] also used the DIC for the analysis of displacement in the crack region, and developed a method for fracture identification in a soft rock. Wolf et al. [16] combined acoustic emission together with the DIC approach for detecting crack development in concrete. This method is not appropriate for the existing structures as the ultrasonic sensors required to be embedded into the concrete structures.

The studies described above were based on standard DIC principles to measure deformation and strain fields of different objects and to investigate existing damage or crack. However, none of them investigated the capability of measuring micro damage or micro-crack using the DIC system. In general, previous investigations employed standard DIC technology with the capability of measuring damage restrained at the macro crack level. In fact, there are a lot of micro cracks on the investigated object surfaces, which cannot be caught by images due to the limited resolution of the current DIC camera equipment. In other words, current DIC technology is in the level for detecting macro crack. This was confirmed by many practical applications and international DIC camera suppliers such as Lavision and Gom Ltd [17].

There is therefore the need to conduct a research into this area of detecting multiscale crack including macro and micro crack. Thereby, this investigation aims to develop the extended digital image correlation (EDIC) method for extending the capability of current DIC from measurement for strain field and macro cracks to detecting micro cracks to improve damage assessment level. It should be noticed that previous investigations [11-15] mentioned the same term of extended digital image correlation, but none of them reported the same EDIC method of detecting multiscale cracks introduced by this paper.

To be able to detect micro cracks in concrete, the EDIC technology is developed using a distance transformation algorithm (DTA), which can computationally detect multiscale cracks in damaged region. Comparing the calculation of the EDIC to scanned images with correlation analysis, multiscale critical damage strains are determined for detecting multiscale crack in concrete. The calculation of correlation can be involved in determination of critical damage strains. The multiscale critical damage strains are a range of strain values at which the cracks with different scales will initiate and propagate. Detecting multiscale cracks will help to identify and monitor damage zones in concrete, which are required in assessing the damage level.

\section{Experimental Work}

A two-dimensional standard DIC camera system (Imager E-Lite 5M Camera - Lavision) is used to observe and record a series of images of a number of unreinforced concrete beam 
specimens subjected to four point bending. The standard DIC is used to analyze the recorded images taken from the surface of investigated concrete beams to derive a deformation field of concrete beam. The proposed EDIC utilizes the extracted data from the camera images including surface coordinates $(x, y)$, displacement fields (dx, dy), strains $\varepsilon(x, y)$ and maximum principal strain $\varepsilon_{\max }$ to determine potential damage area then detect cracks on the surfaces of concrete beams. A range of critical damage strain $\varepsilon_{c}$ is used to establish multiscale crack models including macro and micro cracks. A number of concrete beam samples with two different sizes, $100 \times 100 \times 500 \mathrm{~mm}$ and $100 \times 100 \times 250 \mathrm{~mm}$, is tested in the laboratory. Fig. 1 shows the set up for testing concrete beams under four-point bending.

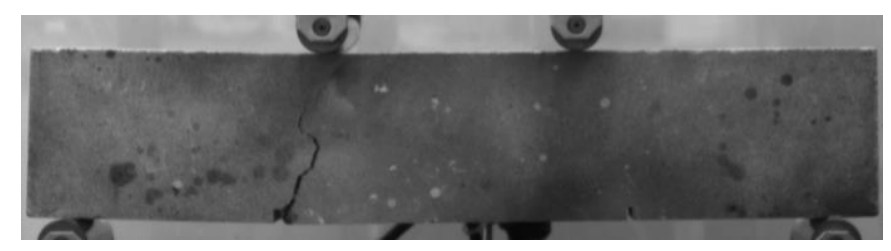

Fig. 1. A concrete beam under-four point bending

As the DIC is an optical measurement technology and only the visible changes in images can be tracked, proper preparation on the specimens is required for camera measurement [17, 18]. A Zwick universal tensile test machine is used in loading concrete beam specimens. The deformation measurement of the sample is monitored and recorded using the DIC camera. The applied load rate is varied between 8 to $25 \mathrm{~N} / \mathrm{s}$. The failure loads of tested samples are between $7500 \mathrm{~N}$ to $12000 \mathrm{~N}$. Fig. 1 shows a failed concrete beam. Because of the quasi-brittle feature of concrete, progressive crack is not clearly appeared. It can be seen from the Fig. 1 that the crack initiated and propagated from the bottom surface to the top surface of the tested beam. The crack widely opened at the bottom where crack initiated then narrowed as it moves towards the top. Before reaching the top, the crack disappeared as it seems completely closed. Actually, micro cracks exist beyond the visible crack front near the top of the beam although they are not visible.

The EDIC technology carried out further analysis based on the image data for detecting and presenting these micro cracks in the tested concrete beam. These data are sorted in the corresponding image correlation system for producing required information such as deformation and strains in the area which is set using a rectangular boundary. It should be noticed that any out of plane rigid body movements [19], e.g. out of plane rigid body movement from an unstable support must be avoided in camera measurement to obtain a right strain field. 


\section{Digital Image Correlation}

138 The DIC camera system used in this investigation is shown in Fig. 2. During loading process specimens are monitored by this system with a charge couple device (CCD) camera which converts photons to electric charge based on the photoelectric effect. The camera sensor consists of many individual CCD elements that are arranged in a rectangular array. The size of each individual CCD element as a pixle is $3.45 \times 3.45 \mu \mathrm{m}^{2}$. This camera with $15 \mathrm{~Hz}$ frequency and resolution of $2448 \times 2050$ pixel is used to take a series of image over time [17]. The basic principle of DIC method was described by a number of previous researchers, e.g. Sutton et al 2009 and Lecompte et al. 2006 [8, 10], to match or track the same subsets located in the reference image and deformed image for retrieving the full-field displacements. The DIC calculates the average gray scale intensity over the subset in deformed and undeformed images, and to perform the correlation of both images by searching the point that has highest grey scale correlating with the initial position of displacement vector in calculating the observable changes in the sequence of images for deriving a deformation field [9]. Using the data from the deformation field the further quantities such as state of strain at any point on the surface can be obtained. The formulas to calculate the maximum principal strain and the principal strain angle are given by Eqs. 1 and 2 .

154

155

156

$161 \varepsilon_{\max }=\frac{\varepsilon_{x x}+\varepsilon_{y y}}{2}+\sqrt{\frac{\left(\varepsilon_{x x}-\varepsilon_{y y}\right)^{2}}{4}}+\varepsilon_{x y}$

$\tan 2 \theta_{p}=\frac{\varepsilon_{x y}}{\varepsilon_{x x}-\varepsilon_{y y}}$

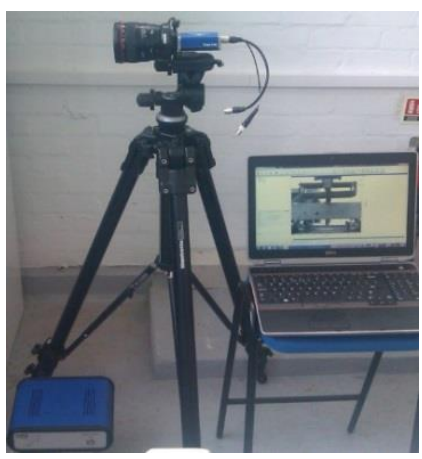

Fig. 2. A DIC camera system

Where, $\varepsilon_{x x}$ and $\varepsilon_{y y}$ are the normal strain in $x$ and $y$ axes respectively, $\varepsilon_{x y}$ is the shear strain and $\theta_{p}$ is the principal strain angle. 


\section{Extended digital image correlation}

The proposed EDIC technology is developed based on a distance transformation algorithm (DTA) to computationally detect multiscale cracks in investigated concrete beams. The data used in the EDIC analysis is supplied by the DIC camera system. At the beginning, the EDIC conducted an undeformed mesh of a beam under zero loading condition. The beam surface is discretized into a set of coordinates $(x, y)$. A basic mesh with $5 \mathrm{~mm}$ grid size is used in this investigation. Then, the EDIC produces a deformed mesh of the beam surface in loading stage. Horizontal and vertical displacements are added into the $(x, y)$ coordinates as $(x+d x, y+d y)$ respectively. Then the EDIC identifies the potential damage zone in which the strain value at each point exceeds the critical damage strain. Fig. 3a shows the damaged zone detected by the EDIC. The length and directions of the arrows in Fig. 3a indicate the amount of strain and direction of crack propagation. It should be noticed that the visible macro crack passed through the path in which each point has maximum length at each group of strain field. The crack propagation in concrete is characterized by appearance of cracks which are perpendicular to the direction of the maximum principal strains. Fig. 3b shows a part of deformed images with cracks. It can be seen clearly from the Figs. $3 a$ and $4 b$ that the relation between the normal to maximum principal strain and crack opening is well described. Finally, the EDIC uses a created mathematical function, distance transform algorithm (DTA) together with the function of maximum stationary point (MSP) to compute the distance transform in determining a crack path along the damaged track in the damaged zone. This damaged track is recognised as a crack path in which every point has an equal distance to the side edges of the damaged zone.

The DTA is used to compute and assign distance transform function in the strain field domain $\Omega$ with a damage region $\Omega^{d}$ shown in Fig. 4. An undamaged region is separated by the boundary $\Omega^{0}$ which is defined as a zero set function. The damage region $\Omega^{\mathrm{d}}$ is defined as a set of all points in which the maximum principal strain value of each point is equal or bigger than the critical damage strain. Whereas, the boundary $\Omega^{\circ}$ defines as a set of points outside of damage region in which the value of maximum principal strain at each point is smaller than critical damage strain. The boundary sets can be denoted mathematically as: $\left\{\Omega^{o}\left|\Omega^{o} \subseteq \Omega, \Omega^{0}\right| \varepsilon_{\max }<\varepsilon_{c}\right\}$. Similarly, the damage region can be denoted as: $\left\{\Omega^{d}\left|\Omega^{d} \subseteq \Omega, \Omega^{d}\right| \varepsilon_{\max } \geq \varepsilon_{c}\right\}$. 


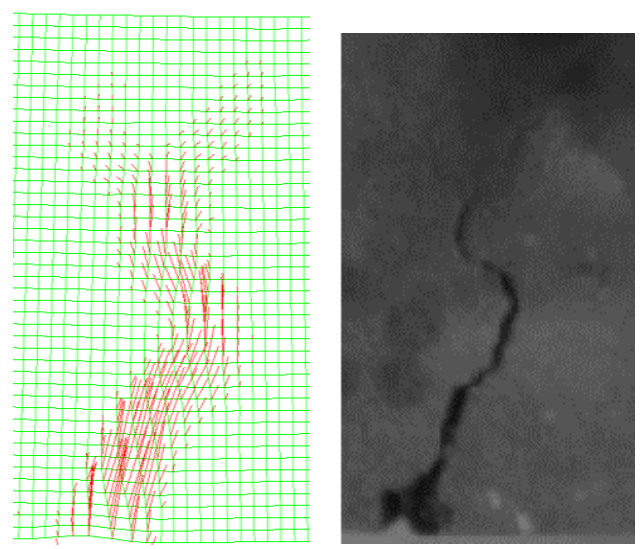

203

Fig. 3. a. A detected damage zone; b. A deformed image with a crack

204

205

206

207

208

209

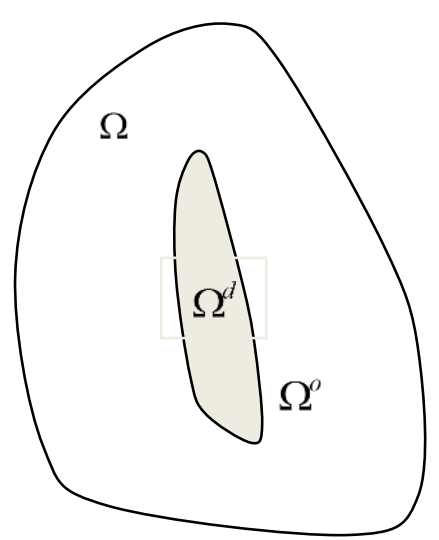

210

Fig. 4. A strain field with a potential damage region

211 The signed distance function $f(x)$ for the sets of points of a boundary and damage region is 212 defined by Eq. 3.

$213 f(x)=\left\{\begin{array}{cl}d(x) & \text { if } x \in \Omega^{d} \\ 0 & \text { if } x \in \Omega^{o}\end{array}\right.$

214 In Eq. 3, the signed distance function for all points on the boundary $\Omega^{0}$ is defined as a zero set 215 function, whereas, the damage region $\Omega^{d}$ represents points signed with distance function $d(x)$, 216 which gives shortest distance from the point $x$ to the boundary $\Omega^{0}$. The signed distance 217 function of all points within the potential damage region $\Omega^{\mathrm{d}}$ can be written into a metric space, 218 and is determined by the distance from a given point $\mathrm{x}$ within the region $\Omega^{\mathrm{d}}$ to the nearest zero 219 point on the boundary $\Omega^{0}$. The value of distance function decreases as the point $x$ approaches 220 the boundary $\Omega^{0}$. 
221 The DTA uses Euclidean distance metric [20] to compute a distance transform of all the points 222 within damage region $\Omega^{\mathrm{d}}$. In two dimensional domain, the Euclidean distance between $\left(\mathrm{x}_{1}, \mathrm{x}_{2}\right)$ 223 and $\left(\mathrm{y}_{1}, \mathrm{y}_{2}\right)$ is given by the Eq. 4.

$d(x)=\sqrt{\left(x_{1}-x_{2}\right)^{2}+\left(y_{1}-y_{2}\right)^{2}}$

225

226

227

$\varepsilon_{i}=\left[\varepsilon_{i 1}, \varepsilon_{i 2} \ldots . \varepsilon_{i n}\right]$

241 The sign result, slope $\left(\varepsilon_{i j}\right)$ between two points can be calculated by Eq. 7.

242 $\operatorname{slope}\left(\varepsilon_{i j}\right)=\operatorname{sign}\left(\operatorname{diff}\left(\varepsilon_{i j}-\varepsilon_{i(j-1)}\right)\right)$

243 The sign function is a mathematical expression defined by the Eq. 8. 
$\operatorname{sign}\left(\varepsilon_{i j}\right)=\left\{\begin{aligned}-1 & \text { if } \varepsilon_{i j}-\varepsilon_{i(j-1)}<0 \\ 0 & \text { if } \varepsilon_{i j}-\varepsilon_{i(j-1)}=0 \\ 1 & \text { if } \varepsilon_{i j}-\varepsilon_{i(j-1)}>0\end{aligned}\right.$

$$
\left\{\begin{array}{l}
\operatorname{slope}\left(\varepsilon_{i j}\right)=1, \operatorname{slope}\left(\varepsilon_{i j}\right) \in R \\
\operatorname{peak}\left(\varepsilon_{i j}\right) \mid \operatorname{slope}\left(\varepsilon_{i j}\right)+\operatorname{slope}\left(\varepsilon_{i(j+1)}\right)=0
\end{array}\right.
$$

247 Where, the $\mathrm{R}$ is an array of sign results in damage region. Finally, all peak values obtained from each row of strain matrix in damage region are used to form the crack path $P$ given by Eq. 10.

$P=\operatorname{plot}\left(\varepsilon_{1 p}, \varepsilon_{i p}, \varepsilon_{m p}\right)$

Where, $\varepsilon_{\mathrm{ip}}$ is the peak value at row i of strain matrix. Eqs. 1 to 10 are used in the developed EDIC programme by Matlab. A flow chart of the EDIC programme can be seen from Fig. 5 . This EDIC algorithm is used in this investigation to computationally detect crack patterns with macro and micro cracks in the following concrete samples.

Fig. 6a shows recorded image of failed concrete sample. Fig. 6b displays the deformed meshes by recorded images given by current DIC camera at the failure points. Fig. $6 \mathrm{~d}$ displays a corresponding strain contour obtained by the DIC algorithm. It can be seen from Fig. $6 \mathrm{~b}$ that current DIC technology can only present the deformed meshes except recorded images. The developed EDIC technology is successfully used to conduct a crack path which can be seen from Fig. 6c. The width of detected crack dictates the amount of strain scaled by the maximum principal strains taken from the strain matrix. The direction of crack growth at each point given in Fig. $6 \mathrm{c}$ is associated with the principal strain vectors. Comparing the EDIC produced crack pattern in Fig. $6 \mathrm{c}$ with the camera images given in Fig. 6a, the EDIC detects the reality of crack paths. In Fig. 6a, the concrete beam lost a large piece of materials at the bottom of beam under bending, which is recorded by a physical image. Obviously, the developed EDIC detects a large piece of materials lost by presented a big crack on the bottom of beam shown in Fig. 6c. Also the EDIC displays the reality of crack opening changes from the bottom to the top of the tested beam. It should be noticed that the failure pattern in Fig. 6a has a crack split when the crack tip approaches the top of the beam. Actually, beyond the split point, there are two brunches of cracks which are not clearly visible on the recorded image in Fig. 6a. It can be seen from Fig. $6 d$ that the strain gradient changes in lower part of strain contour associate 
with the macro crack very well in the lower part of beam, but the upper part of strain contour associated with two invisible brunches of cracks has no clearly visible strain gradient changes. However, these two invisible brunches of cracks are existed and treated as invisible micro cracks in this investigation. The EDIC technology not only captured the split point but also detects two micro crack paths shown in Fig. 6c. Therefore, the developed EDIC supplies an entire failure pattern with macro and micro cracks. More detailed detection of micro cracks will be discussed in the following sections.

In standard DIC analysis the grid level or subset size as the minimum size is used to treat the

Transform data into $A \& d A$ matrices $A$ contains $x \& y$ coordinates. $d A$ contains $\left(x+d x, y+d y, \varepsilon_{m}\right)$

$$
\begin{gathered}
x, y, d x, d y \\
\varepsilon_{m}, \varepsilon_{x x}, \varepsilon_{x y}, \varepsilon_{y y}
\end{gathered}
$$
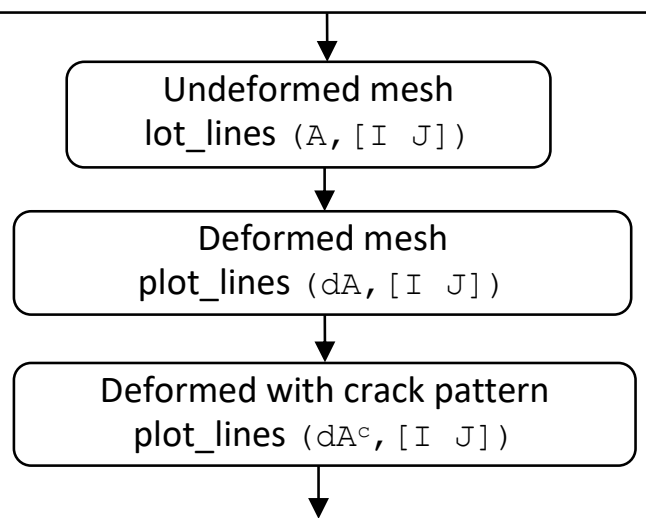

Set all the values bellow the maximum emax to zero

(i.e.to determine potential damage regionby $\varepsilon_{\mathrm{ij}}>=\varepsilon_{\mathrm{c}}$ )

DTA \& MSP used to find the maximum value of the distance transform and create crack paths in potential

damage region $\operatorname{slope}_{k}=\operatorname{sign}\left(\operatorname{diff}\left(\varepsilon_{i j}-\varepsilon_{i(j-1)}\right)\right)$

$$
\left(\text { slope }_{i j}+\text { slope }_{i(j+1)}\right)=0
$$

Correlating monitored damages with image data

$$
C O F=\left[\sum \varepsilon(x, y) \varepsilon^{s}(x, y)\right] \div \sqrt{\left[\sum \varepsilon(x, y)^{2} \varepsilon^{s}(x, y)^{2}\right]}
$$

Plot crack paths by plot_ lines

( $\left.P,\left[\begin{array}{ll}I & J\end{array}\right]\right)$

Fig. 5. The flow chart of the EDIC data of deformation to conduct the strain field. Although the subset size cannot be used directly 
to determine crack size in the EDIC, however, as DIC camera has a fixed resolution, choosing small subset size means more strain points used in conducting an accurate strain field, which ensures the quality of detected cracks by the EDIC. Therefore, it is necessary to investigate the effect of gird level from coarse to refined mesh on detection of crack paths by the developed EDIC, and to identify a converged grid level in which the EDIC can conduct a good crack pattern when comparing to the physically recorded stream of cracks in the investigated concrete beams.
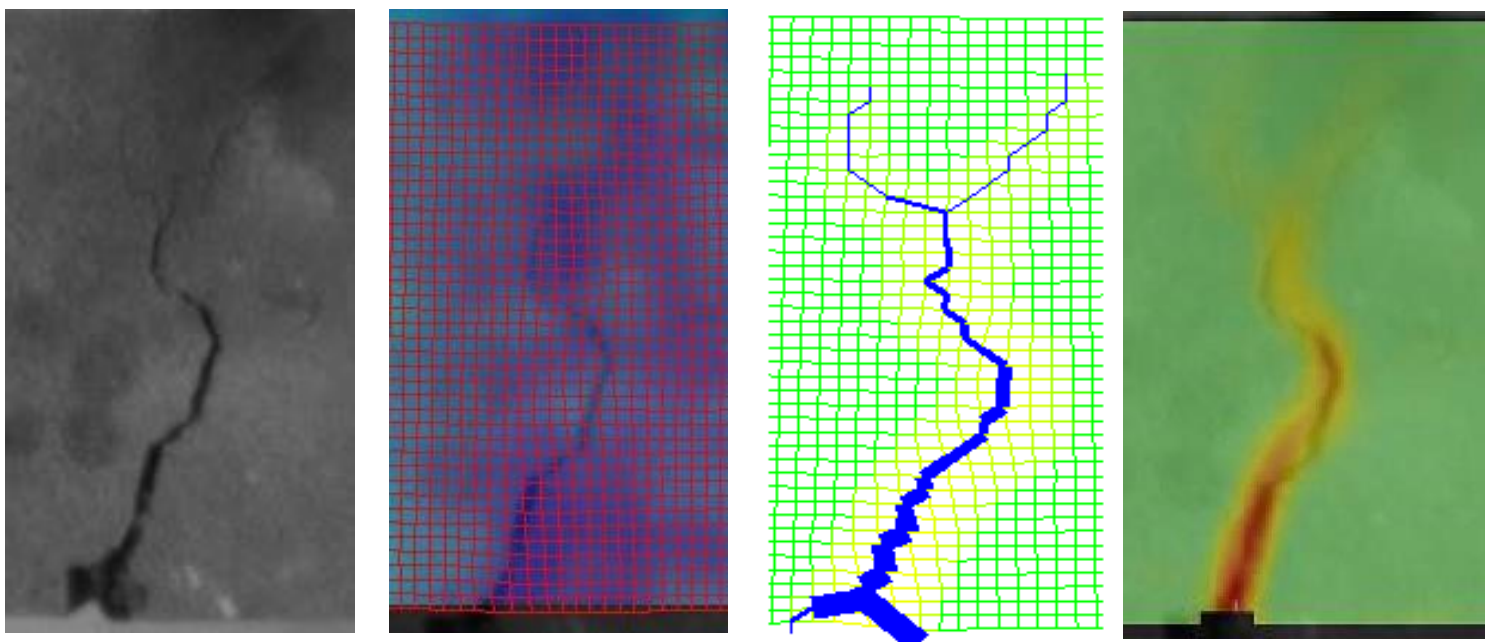

Fig. 6. a. Image with cracks; b. Meshed image; c. Macro and micro crack paths; d. A relevant strain field

Fig. 7 shows detected cracks by the EDIC using different grid levels descended from 2.4 to $0.95 \mathrm{~mm}$. It can be seen from the Fig. 7 that the accuracy of the damaged mode affected by the number or sizes of the subsets on the beam surface, the more discretization of the surface the better the crack approximation detected by the EDIC compared to the image given in Fig. 7. In other word, the smaller grid size has a stronger corresponding than a coarser grid. Therefore, the denser mesh can improve the accuracy of the computational detection of cracks. At the grid level $0.95 \mathrm{~mm}$, the detected crack has an excellent agreement with the image recorded crack path on the beam. It can also be seen from Fig. 7 that the detected crack path at the grid level $1.1 \mathrm{~mm}$ is mostly same with that at the grid level $0.95 \mathrm{~mm}$. This implies that the grid level around $1 \mathrm{~mm}$ is the converged size of the subsets in this investigation.

It should be noted that the camera lens used in this investigation has a fixed focal length (f) of $24 \mathrm{~mm}$. Therefore, for a given lens with a fixed focal length, the measurement distance between the camera and the object determines the magnification. Thus, the size and the number of the subsets will depend on the distance of the camera from the specimens. The closer the camera to the object the smaller the field of view (FoV) of the object hence the 

camera and the specimen is $2.5 \mathrm{~m}$, the smallest grid size given by the camera is $0.95 \mathrm{~mm}$.
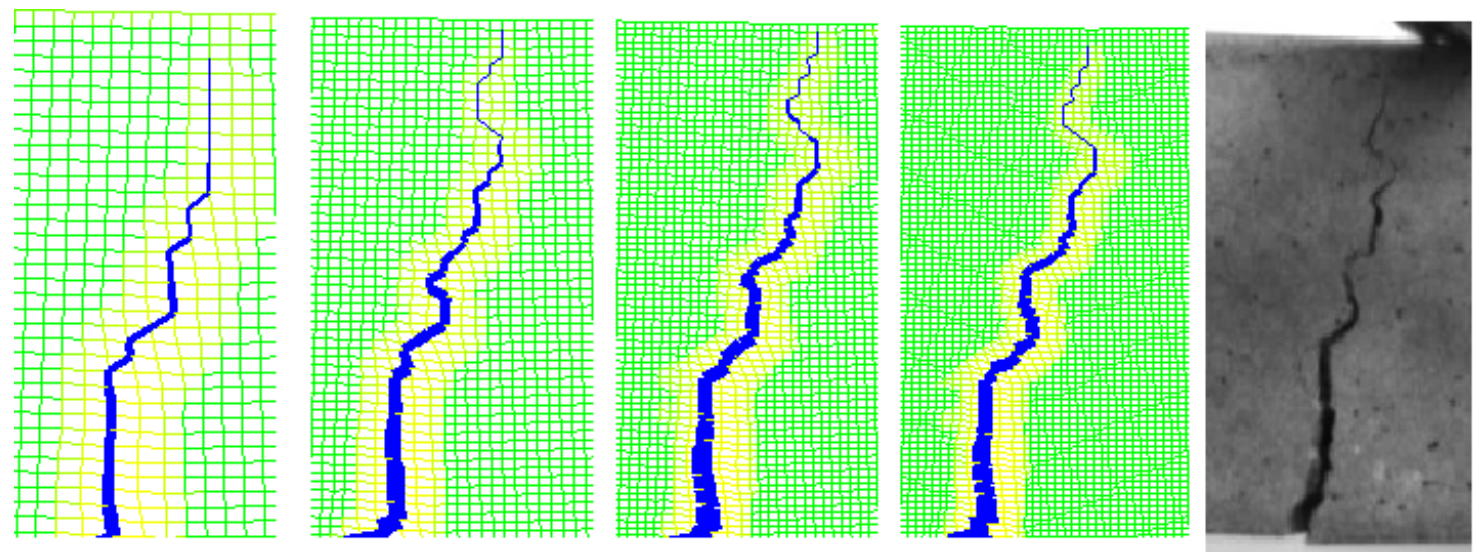

Fig. 7. Detected cracks with different grid levels (unit: $\mathrm{mm}$ ) from left to right: 2.4, 1.4, 1.1, 0.95

In principal, the focal length $f$ of the camera can be determined by Eq. 11 [17].

$$
f=\frac{s s \times d i s}{(F o V+s s)}
$$

340 Where, dis is the measurement distance, ss is the sensor size. Rewriting Eq. 11 gives a calculation of the FoV as shown in Eq. 12.

$342 \quad F o V=\left[\frac{d i s}{f}-1\right] \times s s$

343 The value of the ss of the applied camera is manufactured as $8.4732 \mathrm{~mm}$ and $7.1001 \mathrm{~mm}$ in horizontal and vertical direction respectively [17]. Thus, bring these two values into Eq. 12, the horizontal and vertical FoV at $2.5 \mathrm{~m}$ measurement distance can be obtained as $F_{0} V_{h}=874$ $\mathrm{mm}$ and $F_{O} V_{v}=732 \mathrm{~mm}$ respectively.

347 The characteristic displacement accuracy (CDA) is estimated as 0.05 pixels based on a 348 number of practical engineering measurements [17]. As the camera sensor has a resolution defined by Resh (2448 pixels) and Res $\mathrm{v}_{\mathrm{v}}(2050$ pixels) in horizontal and vertical direction respectively [17], at $2.5 \mathrm{~m}$ distance the applied displacement accuracy (ADA) in horizontal and vertical direction can be calculated by Eq. 13 . 


$$
A D A_{v}=\frac{F o V_{v} \times C D A}{\operatorname{Res}_{v}}==\frac{732 \times 0.05}{2050}=0.01785 \mathrm{~mm}=17.85 \mu \mathrm{m}
$$

355

Thus the strain accuracy (SA) given by the camera in this investigation can be calculated by Eq. 14 as below.

$$
S A_{h}=\frac{A D A_{h}}{F o V_{h}}=\frac{17.85 \times 10^{-3}}{874}=0.204 \times 10^{-4}
$$

$$
S A_{v}=\frac{A D A_{v}}{F o V_{v}}=\frac{17.85 \times 10^{-3}}{732}=0.243 \times 10^{-4}
$$

This calculated strain accuracy given in Eq. 14 is based on $2.5 \mathrm{~m}$ measurement distance. Therefore, the smallest grid size $0.95 \mathrm{~mm}$ can enable the camera to capture the micro change in strain field for computationally detecting micro cracks and predicting crack propagation through detecting invisible micro cracks to visible macro cracks.

\section{Critical damage strain}

The critical damage strain in which a crack forms or develops is one of key issues in this study to enable detecting damage modes in concrete. In general, concrete is a quasi-brittle material and inherently weak in tension [21], and concrete has relatively low strain value [22]. One of objectives in this experimental work is obtaining practical critical damage strains. During loading process as the applied load increases the tensile strain develops on the bottom of the beam. At any point when the critical damage strain is reached a crack is proposed to be formed [23]. The developed EDIC method is also used to determine the value of the critical damage strain. The EDIC uses the data extracted from camera images including coordinates of $(x, y)$, displacement of coordinates ( $d x, d y)$, normal and shear strain $\left(\varepsilon_{x x}, \varepsilon_{x y}, \varepsilon_{y y}\right)$ and the maximum principal strain $\varepsilon_{\max }$ for computational detecting multiscale cracks. The critical damage strain $\varepsilon_{\mathrm{C}}$ is recognized by this investigation as a value of strain which can correlate computationally detected cracks by the EDIC to the image recorded crack path in the potential damage area of the investigated concrete. The multiscale critical damage strains are then used to map the damaged zone which includes macro and micro cracks.

The effect of critical damage strains on computational detecting crack paths is investigated through testing a number of concrete beams. Fig. 8 shows the EDIC detected crack paths in a selected sample. A range of critical damage strains from 0.002 to 0.008 together with subset 
379 size $0.95 \mathrm{~mm}$ is used in the computational detection of four crack patterns for comparing to 380 physically recorded images shown in Fig. 8. A critical damage strain of tested concrete is 381 determined by the calculation of correlation given by Eq. 15.

$C O F=\sum \varepsilon(x, y) \varepsilon^{s}(x, y) / \sqrt{\sum \varepsilon(x, y)^{2} \varepsilon^{s}(x, y)^{2}}$

383

Where, $\varepsilon^{\mathrm{S}}(\mathrm{x}, \mathrm{y})$ is the selected strain array by the EDIC for computational detecting cracks, and $\varepsilon(\mathrm{x}, \mathrm{y})$ is original image strain array supplied by DIC in the damaged region. In fact, when the critical strain of 0.008 is used, the detected crack path does not exactly fit the complete damaged area of the visible cracks in the recorded image. It can be seen from Fig. 8 that the smaller critical strain produces a better crack path that fits into actual recorded crack path. Therefore, the smaller critical strain for instance 0.005 increases the similarity between the computationally detected crack paths and physically recorded one. Further going down, when the critical strain of 0.002 is used, almost possible damaged area is detected, and the EDIC produced crack path reaches the top surface of the beam. In this case, the correlation coefficient calculation given by Eq. 15 is 1 . Actually, the crack in the top area of the beam is a detected micro crack when the critical strain 0.002 is used, which is invisible in the recorded image.

The damage modes detected by the EDIC shown in Fig. 8 using a range of critical damage strains from 0.002 to 0.008 have strong correspondences to the imaged crack patterns of tested concrete samples. This range of critical damage strains of investigated concrete samples is verified by the calculation of correlation together with comparison to images. This range of critical damage strains has a reasonable agreement with previous work by $\mathrm{Lu}$ and $\mathrm{Li}$ [24], which reported that the critical strain of concrete ranges from 0.001 to 0.007 . The values of failure strains at local failure in concrete presented by Ortiz [25] are also within the range of critical damage strains achieved by this study. It should be noticed that the value of critical damage strain used in the EDIC has a scaling effect on the EDIC detection, its details will be discussed by different papers. 

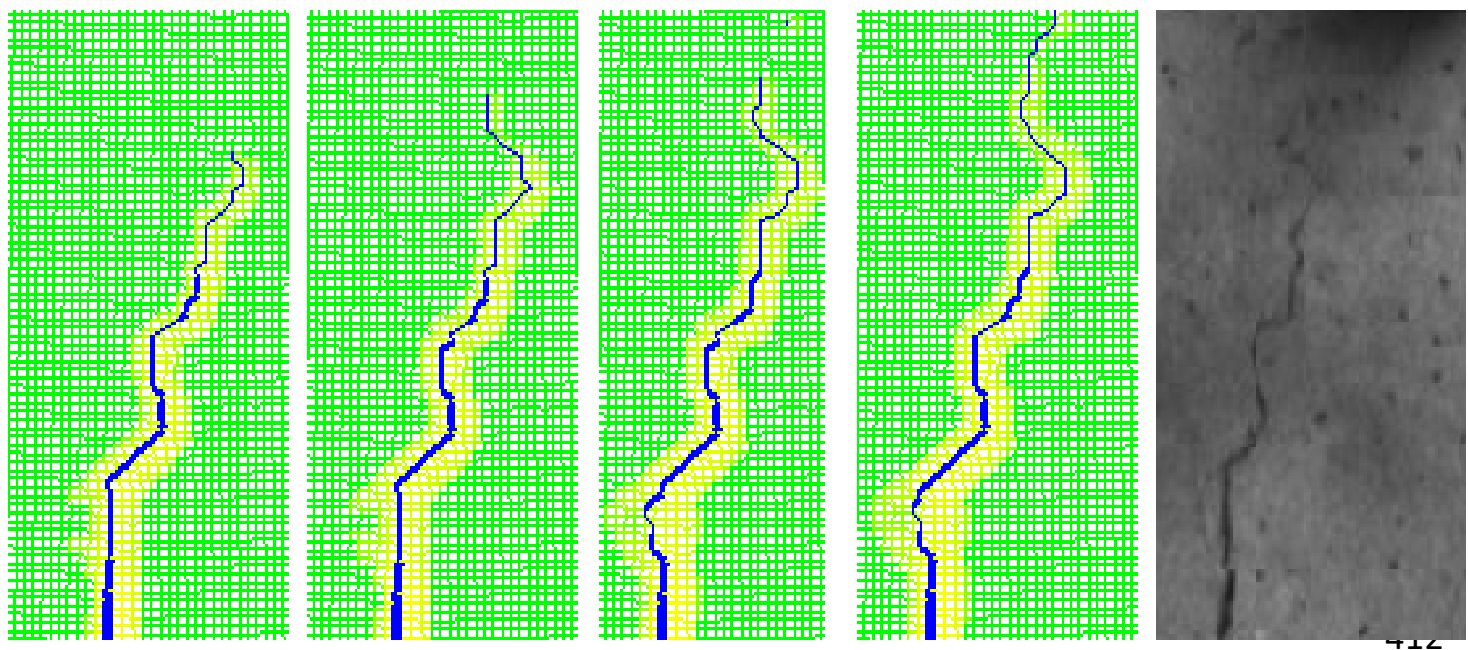

Fig. 8. Predicted crack paths using different critical strains from left to right: $0.008,0.007,0.005,0.002$

\section{Detection of micro cracks}

A range of critical damage strains or multiscale critical damage strain is used to computationally detect multiscale crack in investigated concrete. This is proved by the following examples shown in Fig. 9, which presents the EDIC detected micro cracks together with images recorded at varied loading levels. It should be noted that the increased loading levels are equivalent to the continually recorded image numbers in experimental work. Fig. 9a and $9 \mathrm{~d}$ show tested results of a sample recorded at the images 475 and 480 together with the EDIC detection of multiscale cracks. It is hardly to see a visible crack from Fig. 9a at the image 475. The corresponding strain contour in Fig. 9b shows a bit of visible strain gradient changes at the bottom of beam, however, there are no visible cracks raised at this loading level. As load level increased, a macro crack is clearly recorded at the image 480 shown in Fig. 9d, and corresponding strain contour clearly shows strain gradient changes along the length of the macro crack shown in Fig. 9e. Actually, this macro crack has been detected by the EDIC as a micro crack shown in Fig. 9c at the same loading level of the image 475. In other words, the EDIC detects a micro crack at the image 475, which is not shown in Fig. 9a, and this micro crack is verified by a macro crack recorded at the image 480. Therefore, Fig. 9 proves the capability of the EDIC in detection of micro crack in concrete. The critical damage strain of 0.002 is used in this detection of micro cracks at the image 475. 


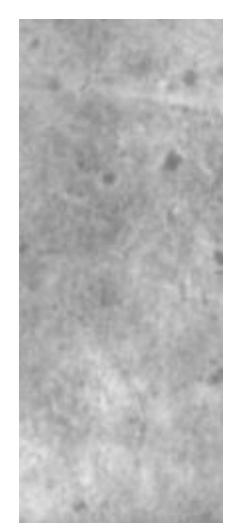

a

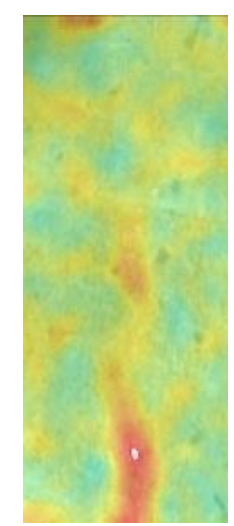

b

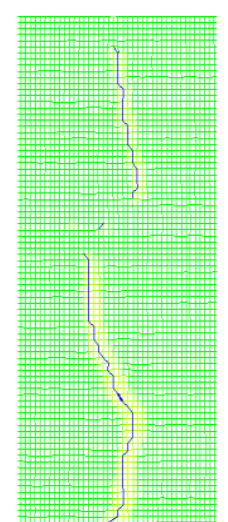

C

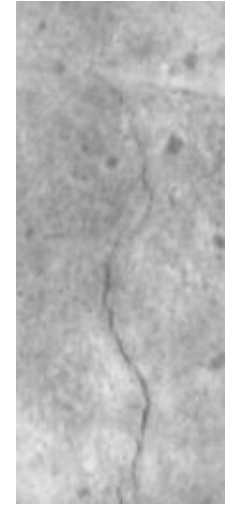

d

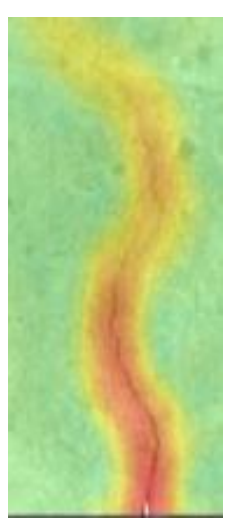

e

Fig. 9. a. The image 475 with no visible crack; b. Strain contour at the image 475; c. The EDIC detected micro crack at the image 475; d. The image 480 with a visible macro crack; e. Strain contour at the image 480.

\section{Conclusion and future work}

The developed EDIC method is successfully used in computational detecting multiscale cracks in concrete beams under bending. The EDIC plays an essential role in mapping the damaged area with macro and micro cracks. The full damage models of tested concrete beams are achieved by the developed EDIC using multiscale critical damage strain. This investigation provides a novel approach of the EDIC in detecting multiscale cracks for damage assessment. The EDIC advantages can essentially help the determination of the damaged area in concrete which needs assessment. This developed EDIC technology has potential to carry out a practical measurement on site to provide a comprehensive damage level of the ageing concrete, and to detect micro crack propagation for ensuring a safe usage of the ageing concrete and planning reparation.

This investigation focuses on the pure concrete beams under flexural loading and finds that the multiscale critical damage strain of investigated concrete is varied from 0.002 to 0.008 . The developed EDIC can also be applied into reinforced concrete for detecting multiscale crack in the future. The currently developed EDIC as a $2 \mathrm{D}$ system can only be used for detecting multiscale crack on the flat surface of objects. The future work will consider extending the developed EDIC technology from a 2D system to a 3D system for mapping damages in the objects with curved surfaces. The current EDIC is able to determine the shape and the length of multiscale cracks, the crack width is detected at macro or micro scale. In the future, the EDIC would be developed to quantitatively compute the width of multiscale cracks. Future work will also include detection of multiscale damages in a wide range of engineering materials. 


\section{Acknowledgements}

The authors would like to acknowledge the BAE Systems surface ships limited, UK for their granting this research and permission to publish this article.

\section{References}

[1] MAPEI, The deterioration of concrete 2015:

http://www.mapei.com/public/IT/linedocument/degrado cls-gb.pdf.

[2] Kiani, K. and Shodja, H. M. (2011). "Prediction of the penetrated rust into the microcracks of concrete caused by reinforcement corrosion." Applied Mathematical Modelling, 35, 25292543.

[3] Jacobsen, J. S., Poulsen, P. N., Olesen, J. F. and Krabbenhoft, K. (2013). "Constitutive mixed mode model for cracks in concrete." Engineering Fracture Mechanics, 99, 30-47.

[4] Dai, Y. Z., Tay, C. J. and Chiang, F. P. (1991). "Determination of the plastic zone by laserspeckle correlation." Experimental mechanics, 31(4), 348-352.

[5] Coburn, D. and Slevin, J. A. (1993). "Development of a digital speckle correlation system for use in the non-destructive testing of advanced engineering ceramics." Key Engineering Materials, 86, 237-244.

[6] Zink, A. G., Davidson, R. W. and Hanna, R. B. (1995). "Strain measurement in wood using a digital image correlation technique." Wood and fiber science, 27(4): 346-359.

[7] Lyons, J. S., Liu, J. and Sutton, M. A. (1996). "High-temperature deformation measurements using digital-image correlation." Experimental mechanics, 36(1), 64-70.

[8] Sutton, M. A., Orteu, J. J. and Schreier, H. W. (2009). "Image correlation for shape, motion and deformation measurements." Springer US 10, 978-0.

[9] Tung, S. H. and Sui, C. H. (2010). "Application of digital-image-correlation techniques in analysing cracked cylindrical pipes." Sadhana, 35(5), 557-567.

[10] Lecompte, D., Vantomme, J. and Sol, H. (2006). "Crack detection in a concrete beam using two different camera techniques." Structural Health Monitoring, 5, 59-68.

[11] Réthoré, J., Roux, S. and Hild, F. (2007). "From pictures to extended finite elements: extended digital image correlation (X-DIC)." Comptes Rendus Mécanique, 335(3), 131-137.

[12] Réthoré, J., François, H. and Stéphane, R. (2008). "Extended digital image correlation with crack shape optimization," International Journal for Numerical Methods in Engineering, 73.2: 248-272. 
[13] Chen, J., Zhang, X., Zhan, N. and Hu, X. (2010). "Deformation measurement across crack using two-step extended digital image correlation method." Optics and Lasers in Engineering, 48(11), 1126-1131.

[14] Réthoré, J., Tinnes, J. P., Roux, S., Buffière, J. Y. and Hild, F. (2008). "Extended threedimensional digital image correlation (X3D-DIC)." Comptes Rendus Mécanique, 336(8), 643649.

[15] Nguyen, T. L., Hall, S. A., Vacher, P. and Viggiani, G. (2011). "Fracture mechanisms in soft rock: identification and quantification of evolving displacement discontinuities by extended digital image correlation." Tectonophysics, 503(1), 117-128.

[16] Wolf, J., Pirskawetz, S. and Zang, A. (2015). "Detection of crack propagation in concrete with embedded ultrasonic sensors." Engineering Fracture Mechanics, 146, 161-171.

\section{[17] Davis L S 2011 User Manual 8}

[18] Robertson, M. A., Borman, S. and Stevenson, R. L. (1999). "Dynamic range improvement through multiple exposures." Image Processing 1999 ICIP 99 Proc. IEEE , 3, 159-163

[19] Sutton, et al. (2008). "The Effect of Out-of-Plane Motion on 2D and 3D Digital Image Correlation Measurements," Optics and Lasers in Engineering, 46, 746-757.

[20] Mathworks, Image Processing Toolbox 2014, User's Guide (R2014a).

[21] Vrech, S. M. and Etse, G. (2012). "Discontinuous bifurcation analysis in fracture energy based gradient plasticity for concrete." International Journal of Solids and Structures, 49, 1294-1303.

[22] Wu, S., Chen, X. and Zhou, J. (2012). "Tensile strength of concrete under static and intermediate strain rates: Correlated results from different testing methods." Nuclear Engineering and Design, 250, 173-183.

[23] Mosley, B., Bungey, J. and Hulse, R. (2007). "Reinforced concrete design to Eurocode 2." 6th edn (Basingstoke: Palgrave Macmillan).

[24] Lu, Y. B. and Li, Q. M. (2011). "About the dynamic uniaxial tensile strength of concretelike materials." International Journal of Impact Engineering, 38, 171-180.

[25] Ortiz, M. (1987). "An analytical study of the localized failure modes of concrete." Mechanics of Materials, 6, 159-174.

[26] Melenka, G. W. and Carey, J. P. (2015). "Evaluation of Fiber Reinforced Cement Using Digital Image Correlation." PloS one, 10(6), e0128644.

[27] Srikar, G., Anand, G. and Prakash, S. S. (2016). "A study on residual compression behavior of structural fiber reinforced concrete exposed to moderate temperature using digital image correlation." International Journal of Concrete Structures and Materials, 10(1), 75-85. 
535 [28] Enfedaque, A., Alberti, M. G. and Galvez, J. C. (2016). "The Influence of Additions in the 536 Use of Glass Fibre Reinforced Cement as a Construction Material." Materials Sciences and 537 Applications, 7(02), 89. 